\title{
Impact of Music on Emotional State and its Management in Youth
}

\author{
Fr Dominic Veigas \\ SVD Secretary \& Treasurer, \\ Certified Gestalt Associate \\ Indore, India.
}

\author{
Ishita Panchal \\ Graduate from Dr. Bhim Rao Ambedkar College, \\ Delhi University \\ Delhi, India
}

\begin{abstract}
At all times we are exposed to a vast variety of sounds consciously or unconsciously, affecting our moods and emotional states. Music has been found to be an effective state management strategy through self-selection and will to alter our mood. The present study explores the effect of unconscious auditory introjections on mood and its management through self-selection. 30 participants were instructed to imagine a past event in their lives expressing specific emotions. The three instructed emotions were anxious, sad and angry. Different expressive music were used including calming, soothing as well as high tempo amped up soundtracks. Immediate verbal responses from the participants during the experiment regarding the study were taken into account. A self-report questionnaire $(m D E S)$ was used to assess the impact of music on mood on the basis of positive and negative emotions experienced. The results showed selfreported positive emotions were significantly higher as compared to negative emotions. No significant differences were found between males and females. The study also explored consciously selected music as a strategy for mood management. Implications and further studies suggestions were also discussed.
\end{abstract}

Keywords: Unconscious, Music, State Management, Emotions, Self-Selected

\section{Introduction}

Humans unconsciously take in a vast amount of information through our various senses.

This Research Paper is on 'Auditory Introjection' and has been authored by Fr Dominic Veigas

This paper has been co-authored by Ishita Panchal, an Intern part of the Global Internship Research Program (GIRP) who graduated from $\mathrm{Dr}$ Bhim Rao Ambedkar College, Delhi University. Delhi. India

Anil Thomas served as a Research Mentor who is a Certified NLP Master Practitioner and Gestalt Therapist. Kainaz Bharucha served as a Statistical Research Adviser who is an Intern part of the Global Internship Research Program (GIRP).This paper has been critically reviewed and proofread by Shaifila Ladhani.

We thank our Trupti Machwe (Our Editor in Chief) and Priya Pawar (Deputy Editor in Chief) for their dedicated time and contribution towards IJNGP.

Correspondence concerning this article should be addressed to

E-mail: office@ijngp
Consciously and unconsciously in our daily lives, we are exposed to various introjections that can alter our state and elicit emotional response thus affecting our behaviour. State is the particular condition at any moment of an individual - our emotions, our thoughts, our sensory register and what we are doing with it. It defines how a person is in a given situation at a given place and time. More than "how" it constitutes a whole lot of variables of "who" a person is at any instant. When we are talking about state, we are specifically referring to internal state, sum total of our psychological and physiological components.

\footnotetext{
Supported by Devashish Polymers

Research and Learning Grants' Partner
} 
Introspecting about the current state and manipulating and managing it helps us in getting the desired behaviour outcome. Depending on how well we are able to manage our state, we can appear to be contracted, reacting and closed, as opposed to grounded, connected and open when managed well. Managing internal states depends a lot on past experience, desired state, immediate as well as future goals and emotional intelligence.

\section{Our Modalities}

Neuro-Linguistic Programming or NLP is a psychological approach using the modeling technique to analyse strategies used by successful individuals for personal goals. It relates thoughts, language, and patterns of behavior learned through experience to specific outcomes. In NLP, VAKOG model elaborates on how people use sensory information with different emphasis. The five sense involved as per VAKOG are,

- VISUAL, It is focused on visual senses. People who are visually oriented most frequently use words like see, look, imagine, examine, inspect, apparently, peek, short-sighted, overview etc.

- AUDITORY, It is focused on auditory senses. Expressions like Quiet, calm, loud, that sounds good, discuss, rumour, hear, speechless, say, whine, something clicked, listen to somebody, clear as a bell, loud and clear, hear somebody out are usually used often by audio oriented people.

- KINESTHETIC, This sense is related to touch, skin and feel. A kinesthetic oriented person might use words like gritty, creepy, heart skips a beat, be on cloud nine, hold on, pull some strings, hand in hand, slip my mind, keep one's feet on the ground, feel secure, ice runs down my spine, foot asleep etc.

- OLFACTORY, It is focused on smell, nose. People who are oriented to this sense often say things like smell, scent, burned, rosy, smoky, mossy, earthy, woody, odourless, perfumed etc.

- GUSTATORY, This channel relates to taste and tongue. Expressions like Juicy, suck, fatty, smack, lick, sip, slobber, taste, burnt, nutty, delicious, dry, oily, spicy, hot, mellow, bitter, sweet, sour, creamy etc.

Individuals give different amounts of emphasis to different senses which reflects in their communication. According to emphasis, people are oriented to different senses which translates to how we perceive the world, how we assess it, how we evaluate it and ultimately elicits a response towards it. This paper is focused on auditory introjections.

\section{Auditory Introjections}

At all times we have our auditory channels on. We may or may not be consciously focusing our attention on audio stimuli but it still affects our state and ultimately our behaviour. The kind of music we listen to, the pitch, loudness, tone all contribute to the unconscious reservoir of thoughts.

The experience that we share with a particular sound also elicits an emotional response from us depending on the previous state that we had when we earlier lived that experience. Unconscious auditory introjections affecting our state can be exemplified in daily life, when we are trying to relax and hear our neighbour having loud party music on it irritates us, however if we are in that party it elevates our mood and makes us groovy.

The fact that we describe sounds in terms of the emotions that they elicit or the mood or frame of mind associated with these sounds for example irritating sounds, calming music, chilling music and so on and so forth, shows the direct effect sounds have on us. Personal experiences, cultural conditioning and personal preferences are 
some of the factors influencing the kind of state any sound brings on in us.

\section{Conscious Management Strategy}

Consciously choosing music to improve one's own internal state or to achieve a desired state outcome represents the individual as being emotionally intelligent. Several studies have shown the favourable impact self-selected music has on our states. Take for instance, a situation where you have to give a speech or a presentation in the office, you play your playlist that you have chosen consciously and instantly you feel calm and composed, ready to ace the presentation. Another way to look at it is through the lens of memories, specific pieces of music elicit specific episodic memories. It might be a song that you heard on your first date or a song that you listened while driving home from your first day at a job or some other good memory associated with a song, now when you hear the same song you instantly get reminded of the memory associated with it and it lifts your mood.

\section{Review of Literature}

There have been a varied range of studies on how music causes neuroendocrine changes. Music induces both psychological and physiological changes in the body. Music therapy is a pragmatic approach to this theoretical framework. It has been shown to be helpful especially for pain and anxiety reduction. (Evans, 2002). Even in the physical rehabilitation field, music is used as a motivator for improvement in motion and motor skills. Different reviews have found music therapy to be facilitating the improvements with regular physical therapy. (Paul \& Ramsey, 2000; Weller \& Baker, 2011). Previous findings have suggested that the kind of music being listened to elicits distinct responses in terms of physiological changes like heartbeat, blood pressure, hormonal secretion and psychological changes like emotional state. Techno-music was found to be related to increased physiological reactions as well as self-reported emotional state, while classical music only showed changes in emotional state (Gerra et al., 1997). In the same vein, Labbe et al. (2007), reported that classical or self-selected music reduces negative emotional states and physiological arousals after an exposure to a stressor as opposed to techno-music or heavy metal music. The study also reflected how music is deeply embedded in youth culture. Despite many studies reflecting the use of music as a self-management strategy, it has received limited attention in sports psychology research. A study on young tennis players on emotional manipulation using music affirmed the above. The findings suggested that consciously selected music elicited the desired state, elevated mood and arousal levels, factors like extramusical associations, desired state, inspirational lyrics and music properties were involved (Bishop et al., 2007). Considering the phenomenon of music eliciting emotions, there has always been dialogues as to whether music elicits genuine emotional responses in listeners (emotivist view) or the listeners merely perceive the emotions elicited by music (cognitivist view). A study by Lundqvist et. al (2009) on this issue involved 32 participants who were measured on selfreported emotions, facial muscle activity and autonomic activity while listening to popular music expressing either sad or happy emotions. The study provided evidence for emotivist position. As the music used was composed specifically for this experiment, the association or past experience to a specific piece of music was also unfounded.

\section{Aim}

The study aims to explore the effect of auditory introjections on the internal state and its management through self-selection. 


\section{Hypotheses}

The following hypotheses are tested in the present study:

- Music has an influence on our states

- Unconscious audio introjections alter our internal states and behaviour

- Consciously selected audio input helps in better state management

\section{Methodology}

Material used: Modified Differential Emotions Scale (mDES) by Frederickson was used to assess the positive and negative emotions during the study. An open-ended survey was used to ask the participants how the perception of their mood states changed throughout the experiment. The survey consisted of 3 questions (How did you feel while imagining the event? How did you feel after listening to the music? And how did you try to focus on the music and away from the past event?).

Scale used: A google form of the mDES was formed consisting of 20 items ( 10 based on positive emotions and 10 based on negative emotions). The scale is purposed to assess the extent to which positive and negative emotions were experienced in a particular time frame.

Participants: The sample consisted of 12 female and 18 male participants of age range 18-24. Purposive sampling was used, the age range was selected considering the importance of music in youth culture and youth being the demographic group using music apps the most.

Procedure : The present study was performed on three groups. For each group, 10 different participants were called on a google meet. The participants were randomly divided into groups for each session. During the google meet, the participants were asked to close their eyes, relax and then were asked to imagine a particular event where they felt anxious (first group) or sad (second group) or angry (third group). After letting them feel the same emotions they were feeling during that particular event, selected music expressing the opposite emotions were played for each group, i.e., weightless by Marconi, Zingaat from Sairat movie and Kun Faya Kun from Rockstar movie, for respective groups. They were instructed to listen carefully as there will be questions about it afterwards, when in fact there were no questions asked. After listening to the music and filling the mDES, they were asked about the difference in their moods before and after listening to the music. The participants were debriefed, the experiment lasted about 30 minutes.

\section{Research Design}

The study was conducted with an experimental design. Purposive sampling method was used to choose participants in the desired age range. For data analysis, qualitative method was used on the openended survey and statistical analysis was done on the raw scores obtained from the self-report mDES questionnaire.

\section{Results}

The scores of positive and negative emotion scores on the mDES are provided in Table 1. For females, the positive emotions were higher than the negative emotions. Similar relation was found among the scores for males and overall in general for the 30 participants. Females showed slightly lower scores on both positive and negative emotion scales, as compared to males. The in-between and amongst groups scores for both positive and negative emotions are shown in fig.1. All of them showed higher scores on the positive scale than on the negative scale.

At the end, during the open-ended survey, the participants responded to having changed mood states from when they were imagining the past event. They reported feeling the same emotions as the time that particular event actually happened. Slowly as they tried to listen to the music carefully and gravitated towards it, they felt a lot 
better, feeling calmer in the case of group 1 and 3, while the group 2 participants felt hyped up and wanting to dance.

Table 1 Means and standard deviations for positive and negative emotion scores for male and female participants as assessed from the mDES.

\begin{tabular}{|l|l|l|l|}
\hline & & $\begin{array}{l}\text { Positive } \\
\text { Emotions }\end{array}$ & $\begin{array}{l}\text { Negative } \\
\text { Emotions }\end{array}$ \\
\hline & Mean & 34.42 & 17.75 \\
\hline FEMALE & $\mathrm{N}$ & 12 & 12 \\
\hline & $\begin{array}{l}\text { Std. } \\
\text { Deviation }\end{array}$ & 3.579 & 3.279 \\
\hline & Mean & 35.17 & 19.72 \\
\hline MALE & $\mathrm{N}$ & 18 & 18 \\
\hline & $\begin{array}{l}\text { Std. } \\
\text { Deviation }\end{array}$ & 3.808 & 7.737 \\
\hline & Mean & 34.87 & 18.93 \\
\hline TOTAL & $\mathrm{N}$ & 30 & 30 \\
\hline & $\begin{array}{l}\text { Std. } \\
\text { Deviation }\end{array}$ & 3.674 & 6.335 \\
\hline
\end{tabular}

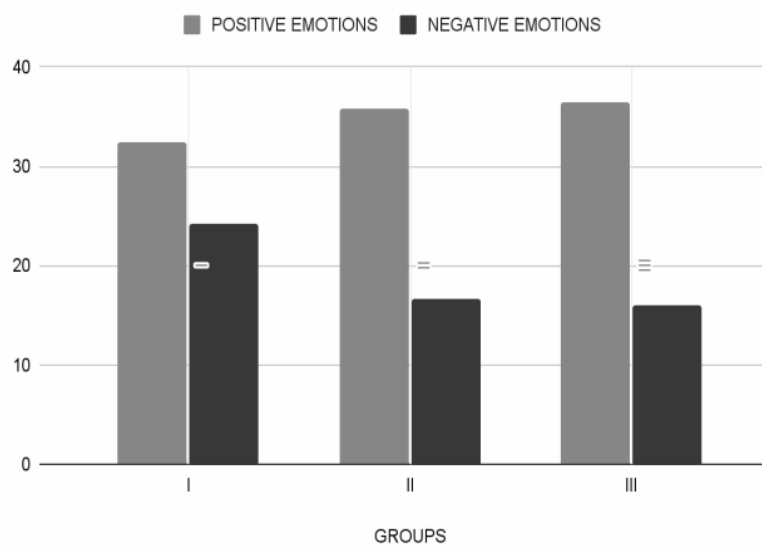

Fig 1 Positive and negative emotion means for each group

NOTE- Each group had 10 participants, the above figure provides the graphical representation of the positive and negative scores as assessed by the mDES questionnaire. Differences among and between groups can also be seen in the figure.

\section{Discussion}

The results of our study support the hypothesis that music impacts the internal state even when exposed to it unconsciously and self-selected music with previous extra musical associations or desired expressions can be used as an emotional management strategy. Listening to the selected music showed significant changes in the emotional responses. The positive emotions score in the self-report questionnaire was higher than the negative emotions score with no significant differences between genders.

Exposure to the stressor event through reliving of the past emotional experience elicited similar responses in the participants during the study. The participants reported feeling anxious, sad or angry during the session as instructed for each group. It can be reflected that the emotions experienced by the participants were cognitive perception of the genuine emotions associated with the original event. This was why they were able to report feeling the same emotions, when the music was played. The participants were not asked to remove themselves from the imagining of the event just yet, to elucidate the relationship between unconscious auditory introjections on mood. The music positively impacted the mood of the participants as can be seen through the figures and table above. Thus confirming our hypothesis that unconscious music has an impact on our emotions. In a similar vein, a study by Eerola (2016) reported even sad music having a positive impact on the listeners' mood and brings them pleasure and comfort. Music designed to have a specific effect on the listener has been found to be most impactful in increasing positive emotions and decreasing negative emotions (McCarty et. al, 1998), thus confirming the findings from our present study. In addition to specifically designed music, the will to alter our mood greatly affects the extent to which we experience happiness while listening to music, as reported in a study by Ferguson et. al (2013).

The music selected was widely popular, especially among youth, owing to their 
respective movies faring extremely well on box office, while for one of the groups, a research backed soundtrack was used which reportedly reduces $65 \%$ anxiety according to a research by mindlab (2019). With years of exposure to music and associated moods through television and movies, people have formed particular cognitive sets with respect to certain types of music (Stratton et. al, 1991). This is reflected in how the expressive emotions in the music affect an individual's emotions or their cognitive perception. For example, when listening to a sad song, we tend to feel down or may get remembered of a sad incident similar to the story expressed in the music. Similarly tempo (high or low) and mode (major or minor) unconsciously causes changes in mood and arousal, as found in a previous study (Hussain et al., 2002).

Lyrical music tracks have also been found to increase attention performance as compared to non-lyrical songs in a previous study (Shih et al., 2016). In the present study, Hindi speaking participants in the third group reflected their understanding of 'Kun Faya Kun' song's lyrics and were able to connect to it more as a result. While the participants in the second group, owing to the fact that they could not understand 'Zingaat' song's marathi lyrics, reported feeling energised just because of the fast beats and high tempo. It can be attributed to the fact that language or words is how people express themselves, understand and communicate with other people or the outside world.

The study has both practical as well as theoretical implications. Music can be explored more as a form of therapy for acute issues like anxiety, depression and mood disorders. Individuals can use it as a management strategy in daily lives to more efficiently manage their emotions and eventually their behaviour. 30 participants were involved in the study, all in the age range of 18-24, i.e. youth and thus cannot be generalised to other demographic groups. Further research can address this issue by studying other demographic and social groups. To elaborate more on the processes during change in emotional states, standardised tests can be explored by the researchers. Physiological responses like heart rate, blood pressure and hormonal change can also be studied to elaborate on the overall changes in the body caused by music.

The study was limited to participants from urban middle class families of a limited age group having ready access to the internet. Hormonal changes were not taken into account due to restriction of resources. Online medium restricted the observations in terms of non-verbal behaviour of the participants. These limitations can be addressed in future studies.

\section{Conclusion}

The present study aimed to elucidate the impact of music on emotional internal state and how self-selected music can be used as a mood management strategy. Using a virtual platform and Google forms, the experiment was conducted in three groups with 10 participants in each group. Modified differential emotions scale $(m D E S)$ was used to assess the effect of music on the participant's mood. The verbal responses of the participants' at the end of the experiment, regarding the difference in their moods before and after listening to the music was also taken into account. The results showed higher levels of positive emotion scores as compared to negative emotion scores on the scale. This proves the hypothesis that music affects our internal state, considering that the participants were instructed to imagine past events involving negative emotions before listening to the music. The study can be explored in a practical paradigm in the form of musical therapy as an intervention as well as a management strategy in daily lives. Future studies can explore different demographic groups to further generalise the present study. 


\section{References}

Bishop, D. T., Karageorghis, C. I., \& Loizou, G. (2007). A Grounded Theory of Young Tennis Players' Use of Music to Manipulate Emotional State. Journal of Sport and Exercise Psychology, 29(5), 584-607. doi:10.1123/jsep.29.5.584

Eerola T, Peltola HR (2016) Memorable Experiences with Sad Music-Reasons, Reactions and Mechanisms of Three Types of Experiences. PLOS ONE 11(6): e0157444. https://doi.org/10.1371/journal.pone.0157444

Evans, D. (2002). The effectiveness of music as an intervention for hospital patients: a systematic review. Journal of Advanced Nursing, 37(1), 8-18. http:// dx.doi.org/10.1046/j.13652648.2002.02052.x

Ferguson, Yuna \& Sheldon, Kennon. (2013). Trying to be happier really can work: Two experimental studies. The Journal of Positive Psychology. 8. 23-33. 10.1080/17439760.2012.747000.

Gerra, G., Zaimovic, A., Franchini, D., Palladino, M., Giucastro, G., Reali, N., ... Brambilla, F. (1998). Neuroendocrine responses of healthy volunteers to 'techno-music': relationships with personality traits and emotional state. International Journal of Psychophysiology, 28(1), 99-111. doi:10.1016/s01678760(97)00071-8

Husain, G., Thompson, W. F., \& Schellenberg, E. G. (2002). Effects of Musical Tempo and Mode on Arousal, Mood, and Spatial Abilities. Music Perception, 20(2), 151-171. doi:10.1525/mp.2002.20.2.151

Koelsch, S., Boehlig, A., Hohenadel, M. et al. The impact of acute stress on hormones and cytokines and how their recovery is affected by music-evoked positive mood. Sci Rep 6, 23008 (2016). https://doi.org/10.1038/srep23008

Labbé, E., Schmidt, N., Babin, J., \& Pharr, M. (2007). Coping with Stress: The Effectiveness of Different Types of Music. Applied Psychophysiology and Biofeedback, 32(3-4), 163-168. doi:10.1007/s10484-007-9043-9

Lundqvist, L.-O., Carlsson, F., Hilmersson, P., \& Juslin, P. N. (2008). Emotional responses to music: experience, expression, and physiology. Psychology of Music, 37(1), 61-90. doi:10.1177/0305735607086048
Magee, W. L., \& Davidson, J. W. (2002). The Effect of Music Therapy on Mood States in Neurological Patients: A Pilot Study. Journal of Music Therapy, 39(1), 20-29. doi:10.1093/jmt/39.1.20 Mindlab (2019). A Study Investigating the Relaxation Effects of the Music Track Weightless by Marconi Union in Consultation with Lyz Cooper. Available at www.britishacademyofsoundtherapy.com/wpcontent/uploads/2019/10/Mindlab-Report-Weightless-Radox$\underline{\text { Spa.pdf }}$

Palmiero, M., Nori, R., Rogolino, C., D'amico, S., \& Piccardi, L. (2016). Sex differences in visuospatial and navigational working memory: the role of mood induced by background music. Experimental Brain Research, 234(8), 2381-2389. doi:10.1007/s00221-016-4643-3

Paul, S., \& Ramsey, D. (2000). Music therapy in physical medicine and rehabilitation. Australian Occupational Therapy Journal, 47(3), 111-118. https://doi.org/10.1046/j.14401630.2000.00215.x

McCraty, Rollin \& Barrios-Choplin, B \& Atkinson, M \& Tomasino, Dana. (1998). The effects of different types of music on mood, tension, and mental clarity. Alternative therapies in health and medicine. 4. 75-84.

Shih, Y.-N., Chien, W.-H., \& Chiang, H. (2016). Elucidating the relationship between work attention performance and emotions arising from listening to music. Work, 55(2), 489-494. doi:10.3233/wor-162408

Stratton, V. N., \& Zalanowski, A. H. (1991). The Effects of Music and Cognition on Mood. Psychology of Music, 19(2), 121-127. doi:10.1177/0305735691192003

Weller, C. M., \& Baker, F. A. (2011). The role of music therapy in physical rehabilitation: a systematic literature review. Nordic Journal of Music Therapy, 20(1), 43-61. https://doi.org/10.1080/08098131.2010.485785

Wheeler, B. L. (1985). Relationship of Personal Characteristics to Mood and Enjoyment after Hearing Live and Recorded Music and to Musical Taste. Psychology of Music, 13(2), 81-92. doi:10.1177/0305735685132002 